\title{
Peripheral anterior chamber depth and screening techniques for primary angle closure disease in community elderly Chinese
}

Qin Luo ${ }^{1,2 \dagger}$, Wenwen Xue ${ }^{1 \dagger}$, Yin Yuan ${ }^{1}$, Chaowei Fu ${ }^{3}$, Jiangnan He ${ }^{1}$, Haidong Zou ${ }^{1,2}$, Xiaowei Tong ${ }^{1}$, Richard K. Lee ${ }^{4}$ and Ling $\mathrm{Ge}^{1,2^{*}}$ (i)

\begin{abstract}
Background: To investigate the distribution of peripheral anterior chamber depth (ACD) and the relationship between peripheral ACD and gonioscopy compared to other ocular parameters for primary angle closure disease (PACD) screening. We performed a population-based survey in Pudong New District of Shanghai, China, in 2011.

Methods: Cross-sectional study. Adults 50 and older were enrolled from a population-based study using cluster random sampling in Pudong New District, Shanghai. Remote ocular screening was performed with digital anterior eye structure photography. Van Herrick measurements were used to evaluate the peripheral ACD, the depth of the peripheral anterior chamber, and corneal thickness $(C T)$, and the ACD to $C T$ ratio was calculated. Subjects with peripheral ACD less than 0.5 CT were made follow-up appointments for clinical examination with gonioscopy. Receiver operating characteristic curves (ROC) were generated to show the performance of different tests in screening for primary angle closure disease (PACD).

Results: Two thousand five hundred twenty-eight adults participated in the study with 91 patients diagnosed with PACD. Two thousand four hundred sixty-three subjects had valid data in the right eye available for analysis. The mean peripheral ACD values for men and women were significantly different: $1.04 \pm 0.46$ (range $0.11-2.93$ ) CT and $0.87 \pm 0.41$ (range $0.12-2.96) C T$ respectively $(t=-4.18 ; P<0.0001)$. Multivariate logistic regression analysis reveals that peripheral ACD declined by $0.31 C T(P<0.0001)$ per diopter of SE and was $0.19 C T(P<0.0001)$ shallower in women than in men $(r 2=0.1304, P<0.0001)$. Peripheral ACD performed best in screening for PACD.
\end{abstract}

Conclusions: Peripheral ACD measurement is recommended for PACD screening in community elderly Chinese.

Keywords: Peripheral anterior chamber depth, Primary angle closure disease, Screening, Chinese

\footnotetext{
* Correspondence: geling99@sina.com

${ }^{+}$Qin Luo and Wenwen Xue contributed equally to this work.

'Department of Ophthalmology, Shanghai Eye Disease Prevention \&

Treatment Center/ Shanghai Eye Hospital, No. 380 Kangding Road, Shanghai

200040, China

${ }^{2}$ Department of Ophthalmology, Shanghai General Hospital, Shanghai Jiao

Tong University School of Medicine, No. 100, Haining Road, Shanghai

200040, China

Full list of author information is available at the end of the article
}

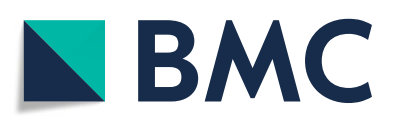

(c) The Author(s). 2020 Open Access This article is licensed under a Creative Commons Attribution 4.0 International License, which permits use, sharing, adaptation, distribution and reproduction in any medium or format, as long as you give appropriate credit to the original author(s) and the source, provide a link to the Creative Commons licence, and indicate if changes were made. The images or other third party material in this article are included in the article's Creative Commons. licence, unless indicated otherwise in a credit line to the material. If material is not included in the article's Creative Commons licence and your intended use is not permitted by statutory regulation or exceeds the permitted use, you will need to obtain permission directly from the copyright holder. To view a copy of this licence, visit http://creativecommons.org/licenses/by/4.0/ The Creative Commons Public Domain Dedication waiver (http://creativecommons.org/publicdomain/zero/1.0/) applies to the data made available in this article, unless otherwise stated in a credit line to the data. 


\section{Background}

Glaucoma is the most common cause of irreversible blindness worldwide, affecting about 67 million people [1-3]. Primary angle closure glaucoma (PACG) is the major type of glaucoma in Asia and is a significant cause of global visual morbidity [4-8]. PACG is higher in Asians than Europeans and Africans, with over $80 \%$ of PACG worldwide cases in Asia. PACG affects approximately $0.75 \%$ adult Asians, doubling in incidence per decade, and $60 \%$ of patients are female. The prevalence rates vary greatly by ethnic region and has been calculated to be approximately $1.10 \%(0.85,1.44)$ in the Chinese population [9]. The rate of blindness caused by PACG is high in mainland China. Random effect model meta-analysis results show the overall blindness rate was $38.3 \%$ [95\%CI $(28.1,49.6 \%)$ ] [10]. A means of detecting those at risk (people with occludable anterior chamber angles) is a prerequisite for a primary angle closure glaucoma prevention program.

Though gonioscopy is recognized as the gold standard for identifying individuals at risk for primary angle closure disease (PACD), this technique requires highly trained personnel and expensive equipment such as a slit lamp (along with gonioscopy lenses), which are in short supply in remote districts in China. Moreover, gonioscopy is relatively subjective, making it less than ideal for comparisons of prevalence between regions [11]. Gonioscopy requires ocular contact examination which may affect the informed consent rate for community screening.

Eyes with PACD tend to have certain biometric characteristics. These include shallow anterior chamber depth (ACD), thick lens, anterior lens position, small corneal diameter and radius of curvature, and short axial length [12-14]. Among these parameters, shallow ACD is known as a key risk factor in most ethnic groups for PACD [15-17].

Some studies suggest that this may not be true for central ACD in East Asian people, where the role of non-pupillary block angle closure in relatively deep anterior chambers has been debated $[15,18]$. Peripheral ACD has proved to be significantly associated with primary angle closure (PAC) and may reflect a nonpupillary block risk factor for PAC to some extent [16].

We performed a population-based survey in Pudong New District of Shanghai, China, in 2011 [19]. 2528 cases were examined and 91 PACD were diagnosed (prevalence rate $3.6 \%$ ), including 6 PACG (prevalence rate $0.24 \%$ ), $9 \mathrm{PAC}$ (prevalence rate $0.36 \%$ ) and 76 primary angle closure suspects (PACS, prevalence rate $3.01 \%$ ) [20]. The goal of this study was to report the distribution of peripheral ACD and its association with age, gender, refraction, and intraocular pressure (IOP) in elderly Chinese people. The relationship between peripheral ACD and gonioscopy was investigated, assessing different methods (peripheral ACD, spherical equivalent $[\mathrm{SE}]$, intraocular pressure [IOP]) for PACD screening in community. Peripheral ACD performed best in screening for PACD.

\section{Methods \\ Investigation place and targets}

This study assessed the Huamu community in Pudong New District of Shanghai. This community has a history of more than 30 years and is home to a stable population. The social and economic levels in the Huamu community are at the average level of Shanghai.

This study was performed in accordance with the Declaration of Helsinki and was approved by the Ethics Committee of Shanghai General Hospital Affiliated to Shanghai Jiaotong University (registration number: 2010 K059). All subjects signed informed consents before being examined.

\section{Sampling}

The detailed study protocol has been described elsewhere [19]. According to the preceding criteria, a total of 3146 people aged 50 and older were enrolled from a population-based study using cluster random sampling in Pudong New District, Shanghai. In the current study, only data from right eyes were analyzed and exclusion criteria include previous cataract surgery and other intraocular operations, ocular trauma, intraocular inflammation, iris dysplasia, atrophic eyeball, and incomplete data.

\section{Investigation procedure}

This research adopted remote screening in the community in combination with subsequent clinical evaluation and diagnosis at a tertiary eye hospital. Subject identities (IDs) were verified and personal information was collected. Remote screening was then performed, including visual acuity, refraction, IOP measurement, slit lamp digital anterior eye structure photography, and digital fundus photography. The collected information was then transmitted to the Shanghai Eye Disease Prevention and Treatment Center through a dedicated network. The ophthalmologists clinically experienced in glaucoma diagnosis reviewed the photographs and gathered data. The investigation team then made an appointment for examination at the Shanghai Eye Disease Prevention and Treatment Center for glaucoma suspects after the preliminary check. Re-examinations included IOP measurement, gonioscopy, perimetry testing by Humphrey automated perimetry, and retinal nerve fiber layer (RNFL) thickness measurement by optical coherence tomography (OCT). 


\section{Ophthalmic assessment}

Visual acuity was measured using a standard illuminated LogMAR (minimum angle of resolution) E chart (Precision Vision, IL, USA), and the presenting visual acuity and the best corrected visual acuity (BCVA) was recorded. The autorefraction data were converted to the spherical equivalent (SE: sphere $+1 / 2$ cylinder).

Digital anterior segment slit lamp photographs were taken in a dark room. Abnormalities of the anterior segment, such as corneal opacity, iris atrophy, pupil size, lens status, presence of glaucomflecken and turbidity of the crystalline lens were recorded. The peripheral ACD around the limbus on the temporal side using an illuminated slit lamp, which casts a clear line on the iris. As described in our previous study [19], peripheral ACD was described as a percentage of corneal thickness at the temporal limbus with the slit beam directed perpendicular to the ocular surface (The brightest, narrowest illumination beam was used. The illumination column was offset from the microscope axis by $40^{\circ}$ ).

Centered by the optic disc and macula, two digital fundus photographs were taken using a digital nonmydriatic fundus camera (CanonCR-DGi, Japan).

All the data collected remotely were transmitted to the Shanghai Eye Disease Prevention and Treatment Center, and the film reading doctors used the Van Herrick method to evaluate the peripheral ACD. Microsoft Paint was used to measure the depth of the peripheral anterior chamber and the corresponding corneal thickness $(\mathrm{CT})$ in the anterior segment photograph, and the ACD to the CT ratio was calculated. Three measurements were carried out and recorded, and the median of 3 readings was used to analyze. A modified Van Herrick grading scheme was used in this study with eight categories $(0,0.05,0.15,0.25,0.35$, $0.45,0.75$, and $\geq 1.0 \mathrm{CT}$ ) instead of the usual five categories $(0,1 / 4,1 / 2,3 / 4,1 \mathrm{CT})$. These values were chosen to give class limits of $0,<0.1,<0.2,<0.3,<0.4$, $<0.5,<1.0$, and $\geq 1.0 \mathrm{CT}$. All subjects with peripheral $\mathrm{ACD}$ less than $0.5 \mathrm{CT}$ were made an appointment for gonioscopy in the tertiary care eye hospital. Microsoft Paint was also used to measure the vertical diameters of the optic cup and optic disk, and vertical cup to disk ratio (VCDR) was calculated. Disk hemorrhage, optic nerve head notching, and other abnormal characteristics in the fundus photograph were recorded.

Gonioscopy was performed with a Goldman onemirror lens (HaggStreit, Bern, Switzerland) at $16 \times$ magnification using a 1-mm-long slit with low ambient illumination to prevent light from irradiating the pupil area. A vertically oriented light beam was used for observing both the superior and the inferior anterior chamber angles and horizontally for the nasal and temporal quadrants. The anterior chamber angle was first evaluated statically, and then dynamically with the lens was performed. The anterior chamber angles were characterized with the Spaeth grading system. One senior doctor did gonioscopy for all participants in the hospital.

SITA-FAST 30-2 mode white-on-white automated perimetry (Humphrey 720, Carl Zeiss Meditec, CA, USA) was performed with refractive correction. RNFL thickness was measured using Cirrus spectral domain HD-OCT (Carl Zeiss Meditec, CA, USA).

\section{Diagnostic definitions}

Glaucoma suspects were identified according to presence of any of the following signs: VCDR $>0.5$ in either eye, VCDR asymmetry $\geq 0.2$, or a neuroretinal rim width reduced to $<0.1 \mathrm{CDR}$ (between 11 and 1 o'clock or 5 and 7 o'clock), optic disk hemorrhage, notching in the optic disc rim or RNFL defects on the superior or inferior temporal near the disc in the fundus photograph, or IOP $\geq 21 \mathrm{mmHg}$ [19].

Glaucoma cases were diagnosed using ISGEO criteria [21]. Glaucoma was identified in accordance with three levels of evidence. The division of glaucoma into PACG versus primary open angle glaucoma (POAG) was based on gonioscopic finding of a narrow angle. PACS was defined as an eye with appositional contact between the peripheral iris and posterior trabecular meshwork [21]. In epidemiological research, a narrow angle has most often been defined as an angle in which $>270^{\circ}$ of the posterior trabecular meshwork (the part which is often pigmented) cannot be seen during a static examination. PAC was regarded as an eye with an occludable drainage angle and features indicating that trabecular obstruction by the peripheral iris have occurred, such as peripheral anterior synechiae (PAS), elevated IOP, iris whorling (distortion of the radially orientated iris fibers), "glaucomfleken" lens opacities, or excessive pigment deposition on the trabecular surface and and the optic disc does not have glaucomatous damage [21].

\section{Statistical analysis}

A database was established with EpiData 3.0 (EpiData Association, Odense, Denmark). Statistical analysis was performed using SAS version 9.1.3 (SAS Inc., NC, USA). The $95 \%$ confidence interval (CI) was calculated assuming a normal distribution. This study first performed univariate logistic regression analysis on the factors influencing peripheral $\mathrm{ACD}$, and then performed multivariate logistic regression analysis to explore the association of age, gender, IOP, and refraction with peripheral ACD. A value of $P<0.05$ was defined as statistically significant. 


\section{Results}

A total of 2528 subjects of the screened 3146 adults participated in the study with valid data, giving a response rate of $80.36 \%$ [20]. Sixty-five cases were excluded in the study because of previous intraocular surgery, ocular trauma, intraocular inflammation, iris dysplasia, atrophic eyeball, and/or incomplete data in right eye. Two thousand four hundred sixty-three subjects had valid data in right eye available for analysis (Fig. 1).

The mean peripheral ACD values for men and women were significantly different: $1.04 \pm 0.46$ (range 0.11-2.93) $\mathrm{CT}$ and $0.87 \pm 0.41$ (range $0.12-2.96$ ) CT respectively $(t=-4.18 ; \quad P<0.0001 ;$ Table 1$)$. Older subjects had shallower peripheral ACD, observed in both men and women and all subjects $(F=4.54 ; P=0.0035$; Table 1$)$, except in the oldest group (80-94 years old). The peripheral ACD between age group 50-60 and age group 70-80 were significantly different.

Univariate logistic regression analysis on demographic factors (age, gender, IOP, SE) influencing peripheral ACD showed that gender $(r=-0.18, P<$ $0.0001)$ and $\mathrm{SE}(r=-0.31, P<0.0001)$ were significantly associated with peripheral ACD (Table 2). Multivariate logistic regression analysis of the association between peripheral ACD and other ocular parameters ( $\mathrm{r} 2=0.1304, P<0.0001)$ revealed that peripheral ACD declined by $0.31 \mathrm{CT}(P<0.0001)$ per diopter of SE and was $0.19 \mathrm{CT}(P<0.0001)$ shallower in women than in men (Table 3).

Totally, 291 subjects had peripheral ACDs less than $0.5 \mathrm{CT}$ in right eyes. The mean peripheral ACD values for men and women with peripheral ACD less than $0.5 \mathrm{CT}$ in right eyes were $0.37 \pm 0.08$ (range 0.11-0.49) CT and $0.35 \pm 0.09$ (range 0.12-0.49) CT respectively $(t=-1.46 ; \quad P=0.1464 ;$ Table 4$)$. The mean peripheral ACD in different age groups was not significantly different.

A total of 291 subjects with peripheral ACD less than $0.5 \mathrm{CT}$ were made an appointment for gonioscopy in the tertiary eye hospital and 91 PACD subjects were identified, including 6 PACG, 9 PAC and 76 PACS.

As gonioscopy was the standard examination for identifying PACD, we compared the efficacy of three screening indexes (peripheral ACD, SE, peripheral ACD combined with SE) with gonioscopy in 291 subjects for identifying PACD (Fig. 2). Using peripheral ACD as screening index achieved sensitivity of 0.618 , specificity of 0.862 , positive predictive value of 0.498 and Youden index of 0.48 with a cut-off value of $0.305 \mathrm{CT}$. Using SE as screening index was not significant and the combination of peripheral ACD and SE did not improve screening efficiency (Table 4).

When a modified Van Herrick grading scheme was used to grade the peripheral ACD, the sensitivity, specificity, positive predictive value and Youden index were

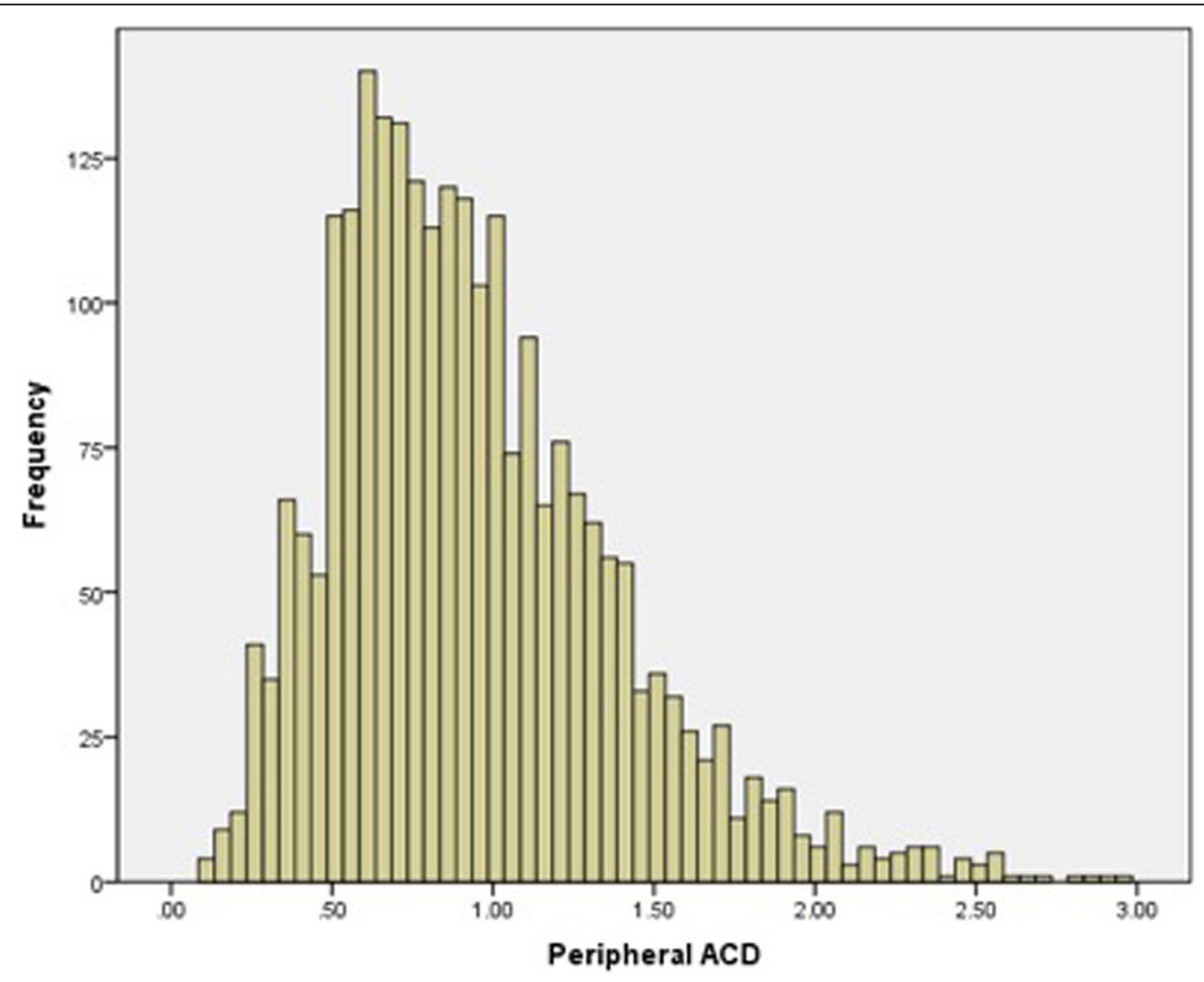

Fig. 1 Histogram showing distribution of peripheral ACD (mean, 0.94 CT; standard deviation, 0.44 CT) in 2463 right eyes 
Table 1 Characteristic of peripheral ACD in right eyes

\begin{tabular}{|c|c|c|c|c|c|c|c|c|}
\hline & $n$ & Mean \pm SD & Median & 25th Percentile & 50th Percentile & 75th Percentile & Missing & Range \\
\hline \multicolumn{9}{|c|}{ Men (age) } \\
\hline $50-60$ & 368 & $1.06 \pm 0.47$ & 1.00 & 0.72 & 1.00 & 1.37 & 0 & $0.18-2.93$ \\
\hline $60-70$ & 398 & $1.02 \pm 0.46$ & 0.94 & 0.69 & 0.94 & 1.30 & 0 & $0.11-2.81$ \\
\hline $70-80$ & 218 & $1.00 \pm 0.44$ & 0.94 & 0.67 & 0.94 & 1.24 & 0 & $0.21-2.60$ \\
\hline $80-94$ & 52 & $1.11 \pm 0.59$ & 1.02 & 0.65 & 1.02 & 1.29 & 0 & $0.28-2.72$ \\
\hline All & 1036 & $1.04 \pm 0.46$ & 0.97 & 0.69 & 0.97 & 1.30 & 0 & $0.11-2.93$ \\
\hline \multicolumn{9}{|c|}{ Women (age) } \\
\hline $50-60$ & 610 & $0.91 \pm 0.40$ & 0.85 & 0.63 & 0.85 & 1.11 & 0 & $0.12-2.65$ \\
\hline $60-70$ & 497 & $0.84 \pm 0.40$ & 0.79 & 0.54 & 0.79 & 1.08 & 0 & $0.13-2.33$ \\
\hline $70-80$ & 245 & $0.84 \pm 0.44$ & 0.75 & 0.54 & 0.75 & 1.06 & 0 & $0.17-2.85$ \\
\hline 80-94 & 75 & $0.94 \pm 0.52$ & 0.78 & 0.60 & 0.78 & 1.18 & 0 & $0.26-2.96$ \\
\hline All & 1427 & $0.87 \pm 0.41$ & 0.81 & 0.59 & 0.81 & 1.09 & 0 & $0.12-2.96$ \\
\hline \multicolumn{9}{|c|}{ Men and Women (age) } \\
\hline $50-60$ & 978 & $0.97 \pm 0.43$ & 0.90 & 0.65 & 0.90 & 1.21 & 0 & $0.12-2.93$ \\
\hline $60-70$ & 895 & $0.92 \pm 0.43$ & 0.86 & 0.60 & 0.86 & 1.16 & 0 & $0.11-2.81$ \\
\hline $70-80$ & 463 & $0.91 \pm 0.45$ & 0.83 & 0.60 & 0.83 & 1.15 & 0 & $0.17-2.85$ \\
\hline $80-94$ & 127 & $1.01 \pm 0.56$ & 0.87 & 0.61 & 0.87 & 1.26 & 0 & $0.26-2.96$ \\
\hline All & 2463 & $0.94 \pm 0.44$ & 0.87 & 0.63 & 0.87 & 1.19 & 0 & $0.11-2.96$ \\
\hline
\end{tabular}

SD Standard deviation

presented in Table 5. The Youden index was greatest with the cut point of the peripheral ACD grade as 0.3 (Table 5).

\section{Discussion}

In this population-based study of Chinese residents of Shanghai, 291 subjects were observed to have a peripheral ACD less than $0.5 \mathrm{CT}$ in their right eyes. Among these subjects, $91(31.27 \%, 91 / 291)$ subjects were diagnosed as having PACD. In this study, digital remote screening and clinical re-examination for a diagnosis were used at a tertiary eye hospital. Information on all subjects was first collected and analyzed remotely, including visual acuity examination, refraction, IOP measurement, digital anterior slit lamp eye structure photography and digital fundus photography. After this information transmitted to the Shanghai Eye Disease Prevention and Treatment Center, glaucoma suspects were asked permission to proceed with additional and confirmatory reexamination [20].

In this study, the peripheral ACD in 2463 subjects were $0.94 \pm 0.44 \mathrm{CT}$, and $11.8 \%$ subjects had reported a peripheral ACD $<0.5 \mathrm{CT}$. The population distribution of peripheral ACD has been studied in Europe, North America, and Asia [22-24].

Based on peripheral anterior chamber depth, Van Herrick classification has been used to detect angle closure glaucoma (ACG) eyes [25]. This method has proved to be effective and suitable for glaucoma screening. However, Van Herrick's classification only had five categories $(0,1 / 4,1 / 2,3 / 4,1$ CT). The Van Herrick's classification has been suggested to have low sensitivity and specificity to detect patients with ACG [11]. Studies in southern India, and Greenland reported suboptimal performance of this test in screening for PAC

Table 2 Multivariate analysis of the association between peripheral ACD and other parameters

\begin{tabular}{lllll}
\hline Variable & $\begin{array}{l}\text { Nonstandardized Regression } \\
\text { Coefficient }(95 \% \mathrm{Cl})\end{array}$ & $\begin{array}{l}\text { Standardized } \\
\text { Regression } \\
\text { Coefficient }\end{array}$ & $\begin{array}{l}\text { Variance } \\
\text { Inflation } \\
\text { Factor }\end{array}$ \\
\hline Intercept & $1.34(1.17,1.51)$ & 0.00 & 0.00 & $\begin{array}{l}P \text { value } \\
\text { Age } \\
\text { Gender }\end{array}$ \\
IOP & $-0.00(-0.00,0.00)$ & -0.02 & 1.02 & 0.0001 \\
SE & $-0.17(-0.20,-0.13)$ & -0.19 & 1.01 & $<.0001$ \\
\hline
\end{tabular}


Table 3 Peripheral ACD values and SE in 291 subjects with peripheral ACD less than 0.5 CT in right eyes

\begin{tabular}{|c|c|c|c|c|c|c|c|}
\hline \multirow[t]{2}{*}{ Gender } & \multirow[t]{2}{*}{ Age } & \multicolumn{3}{|c|}{ Peripheral ACD } & \multicolumn{3}{|l|}{ SE } \\
\hline & & $\mathrm{n}$ & Mean \pm SD & Range & $\mathrm{n}$ & Mean \pm SD & Range \\
\hline \multirow[t]{5}{*}{ Men } & $50-60$ & 25 & $0.36 \pm 0.09$ & $0.18-0.49$ & 25 & $0.93 \pm 1.27$ & $-1.00-4.625$ \\
\hline & $60-70$ & 28 & $0.37 \pm 0.08$ & $0.11-0.47$ & 28 & $1.00 \pm 1.61$ & $-5.375-3.75$ \\
\hline & $70-80$ & 19 & $0.39 \pm 0.08$ & $0.21-0.48$ & 16 & $0.09 \pm 5.03$ & $-17.375-4.25$ \\
\hline & 80-94 & 5 & $0.43 \pm 0.09$ & $0.28-0.49$ & 4 & $1.22 \pm 2.12$ & $-1.625-3.50$ \\
\hline & subtotal & 77 & $0.37 \pm 0.08$ & $0.11-0.49$ & 73 & $0.79 \pm 2.67$ & $-17.375-4.625$ \\
\hline \multirow[t]{5}{*}{ Women } & $50-60$ & 61 & $0.37 \pm 0.10$ & $0.12-0.49$ & 60 & $0.78 \pm 1.03$ & $-3.00-3.75$ \\
\hline & $60-70$ & 91 & $0.35 \pm 0.09$ & $0.13-0.49$ & 90 & $0.77 \pm 1.53$ & $-8.25-3.875$ \\
\hline & $70-80$ & 53 & $0.35 \pm 0.09$ & $0.17-0.49$ & 49 & $0.96 \pm 1.65$ & $-4.625-4.125$ \\
\hline & 80-94 & 9 & $0.33 \pm 0.07$ & $0.26-0.46$ & 6 & $2.08 \pm 1.43$ & $0.50-4.25$ \\
\hline & subtotal & 214 & $0.35 \pm 0.09$ & $0.12-0.49$ & 205 & $0.85 \pm 1.44$ & $-8.25-4.25$ \\
\hline \multirow[t]{5}{*}{ All } & $50-60$ & 86 & $0.37 \pm 0.09$ & $0.12-0.49$ & 85 & $0.82 \pm 1.10$ & $-3.00-4.625$ \\
\hline & $60-70$ & 119 & $0.35 \pm 0.09$ & $0.11-0.49$ & 118 & $0.82 \pm 1.55$ & $-8.25-3.875$ \\
\hline & $70-80$ & 72 & $0.36 \pm 0.09$ & $0.17-0.49$ & 65 & $0.74 \pm 2.84$ & $-17.375-4.25$ \\
\hline & $80-94$ & 14 & $0.37 \pm 0.09$ & $0.26-0.49$ & 10 & $1.74 \pm 1.68$ & $-1.625-4.25$ \\
\hline & total & 291 & $0.36 \pm 0.09$ & $0.11-0.49$ & 278 & $0.84 \pm 1.84$ & $-17.375-4.625$ \\
\hline
\end{tabular}

$[26,27]$. Therefore, in this study, a modified Van Herrick grading scheme was performed with eight categories $(0$, $0.05,0.15,0.25,0.35,0.45,0.75$, and $\geq 1.0$ CT) instead of the usual five categories. These values were chosen to give class limits of $0,<0.1,<0.2,<0.3,<0.4,<0.5,<1.0$, and $\geq 1.0 \mathrm{CT}$. This technique has been demonstrated to be valid in other studies [28-30].

Our research shows that the peripheral ACD declines by $0.31 \mathrm{CT}$ per diopter of SE and was $0.19 \mathrm{CT}$ shallower in women than in men. Our findings agree with previous studies that women are more likely to have a shallower peripheral ACD [31]. Similar to other reports, our results are consistent with hyperopic patients likely to have shallower ACDs [32].

The present study used three different indexes (SE, peripheral $\mathrm{ACD}, \mathrm{SE}$ combined with peripheral $\mathrm{ACD}$ ) to investigate the most efficient way for PACD screening. The independent SE as the screening index for PACD was not statistically observed and did not significantly improve the screen capability of peripheral ACD for PACD, too. On the basis of these findings, only peripheral ACD measurement should be used to PACD screening in community elderly Chinese.
In our research, when peripheral ACD was $<0.3 \mathrm{CT}$, Youden index performed best in all groups and the positive predictive value was 0.703 , however, a higher sensitivity is needed for effective community screening. The use of a CT cut off value of 0.4 would achieve much higher sensitivity (0.846). Our findings disagree with those from North America, Greenland, and Australia, which reported a cut-off value of 0.25 CT $[26,28,33]$. The reasons may be that the pathogenisis of angle closure is different in different population and the assessment method of peripheral ACD in this study is different from that in other studies. Similarly, Foster et al. also reported the augmented Van Herrick scheme offers enhanced performance in detection of established PACG [29].

A limitation of this study is that only subjects with peripheral $\mathrm{ACD}<0.5 \mathrm{CT}$ were asked to undergo gonioscopy, which may give rise to a potential cause of bias. Three major rationales were used for our screening method design. First, according to Van Herrick, a subject with a peripheral $\mathrm{ACD} \geq 0.5 \mathrm{CT}$ would have a very low probability of having angle closure [25]. Second, there was a study in Australia, in which goniscopy was

Table 4 AUC of different ROC curve and cut-off value

\begin{tabular}{lllllll}
\hline ROC curve & AUC $(95 \% \mathrm{Cl})$ & Cut-off Value & Se & Sp & PV+ & Youden Index \\
\hline peripheral ACD + SE & $0.786(0.726,0.847)$ & peripheral ACD $=0.360$ SE $=2.120$ & 0.809 & 0.670 & 0.527 & 0.479 \\
peripheral ACD & $0.777(0.715,0.839)$ & peripheral ACD $=0.305$ & 0.618 & 0.862 & 0.498 & 0.480 \\
SE & $0.547(0.476,0.619)$ & SE $=1.188$ & 0.652 & 0.468 & 0.346 & 0.120 \\
\hline
\end{tabular}

AUC Area under curve, Se Sensitivity, Sp Specificity, $P V+$ Positive predictive value 


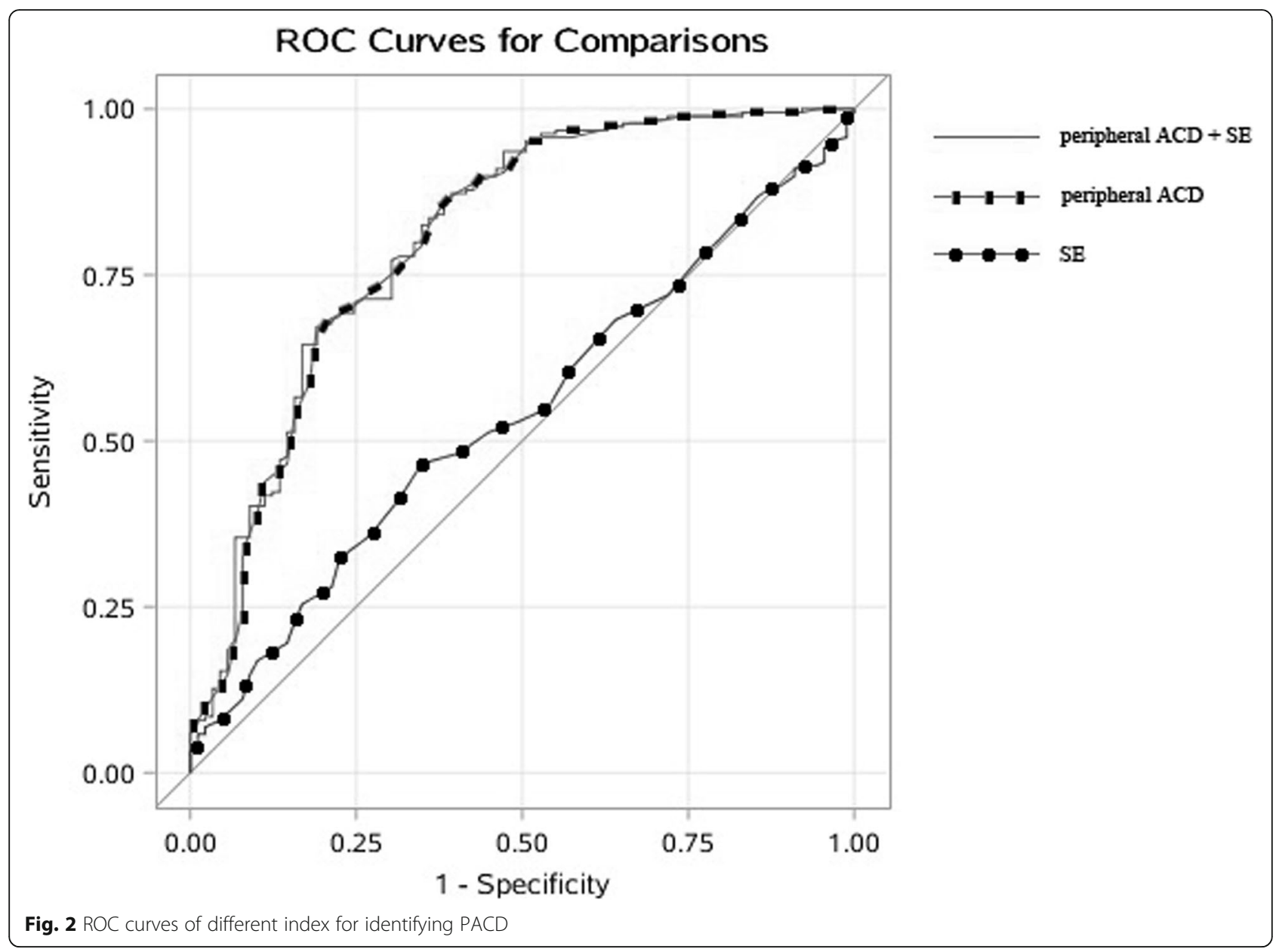

carried out only with peripheral $\mathrm{ACD} \leq 0.3 \mathrm{CT}$ [28]. Third, Foster et al. performed goniscopy on subjects and indicated that in subjects witth peripheral $\mathrm{ACD} \geq 0.5 \mathrm{CT}$, no patients had closed angles. Moreover, when the cut off of $<0.3$ CT with peripheral ACD was used in this study, the Youden index was still not particularly high, which called a measure with a higher sensitivity for community screening of glaucoma. Therefore, a peripheral $\mathrm{ACD}<0.5 \mathrm{CT}$ is likely to be a relatively cost-effective index for glaucoma screening, although we do not recommend that only the estimation of peripheral ACD be used instead of gonioscopic examination in patients suspected of glaucoma.

Table 5 The efficacy of identifying PACD with different peripheral ACD

\begin{tabular}{lllll}
\hline Peripheral ACD grade & Se & Sp & PV+ & Youden Index \\
\hline$<0.2$ & 0.154 & 0.995 & 0.933 & 0.100 \\
$<0.3$ & 0.571 & 0.890 & 0.703 & 0.403 \\
$<0.4$ & 0.846 & 0.475 & 0.423 & 0.291 \\
$<0.5$ & 1.000 & 0.000 & 0.313 & 0.000 \\
\hline
\end{tabular}

Se Sensitivity, Sp Specificity, $P V+$ Positive predictive value

\section{Conclusions}

Peripheral ACD measurement is recommended for PACD screening in community elderly Chinese. Further research is still required for finding out the most efficient screening technique for primary angle closure disease in China.

\section{Abbreviations}

ACD: Anterior chamber depth; SE: Spherical equivalent; IOP: Intraocular pressure; CT: Corneal thickness; ROC: Receiver operating characteristic curves; PACD: Primary angle closure disease; PACG: Primary angle closure glaucoma; POAG: Primary open angle glaucoma; RNFL: Retinal nerve fiber layer; OCT: Optical coherence tomography; VCDR: Vertical cup to disk ratio; PAS: Peripheral anterior synechiae

\section{Acknowledgements}

Not applicable.

\section{Authors' contributions}

QL, WWX and LG were major contributors in writing the manuscript. CWF analyzed data. QL designed the monograph plan. WWX coordinated the data analysis. LG designed the general introduction. YY, JNH, XWT, and HDZ collected the data. LG and RKL made the English translation. All authors read and approved the final manuscript.

\section{Funding}

Project "Exploration of remote screening mode of glaucoma in community (2010281)" provided financial support for the design of the study and 
collection, analysis, and interpretation of data and in writing the manuscript. Project "Feasibility study and construction of remote screening system model of eye disease in community (20114007)" from Shanghai Municipal Health Bureau provided financial support for the design of the study. Project "Opportunistic screening for primary glaucoma in community and establishment and evaluation of the remote screening technology (SHDC12014204)" from Shanghai Shenkang Hospital Development Center provided financial support in the interpretation of data. R.K. Lee is supported by the Walter G. Ross Foundation.

\section{Availability of data and materials}

The datasets used and analysed during the current study are available from the corresponding author on reasonable request.

\section{Ethics approval and consent to participate}

This study was performed in accordance with the Declaration of Helsinki and was approved by the Ethics Committee of Shanghai General Hospital Affiliated to Shanghai Jiaotong University (registration number: 2010 K059).

\section{Consent for publication}

Not Applicable.

\section{Competing interests}

The authors declare that they have no competing interests.

\section{Author details}

'Department of Ophthalmology, Shanghai Eye Disease Prevention \& Treatment Center/ Shanghai Eye Hospital, No. 380 Kangding Road, Shanghai 200040, China. ${ }^{2}$ Department of Ophthalmology, Shanghai General Hospital, Shanghai Jiao Tong University School of Medicine, No. 100, Haining Road, Shanghai 200040, China. ${ }^{3}$ Department of Social Medicine, School of Public Health, Key Laboratory of Public Health Safety, NHC Key Laboratory of Health Technology Assessment, Fudan University, Shanghai 200032, China. ${ }^{4}$ Bascom Palmer Eye Institute, 900 N.W. 17th Street, Miami, FL 33136, USA.

Received: 10 February 2020 Accepted: 20 August 2020

Published online: 28 August 2020

\section{References}

1. Quigley HA. Number of people with glaucoma worldwide. Br J Ophthalmol. 1996;80(5):389-93.

2. Thylefors B, Negrel AD, Pararajasegaram R, et al. Global data on blindness. Bull World Health Organ. 1995;73(1):115-21.

3. Wong TY, Loon SC, Saw SM. The epidemiology of age related eye diseases in Asia. Br J Ophthalmol. 2006;90(4):506-11.

4. Foster PJ, Baasanhu J, Alsbirk PH, et al. Glaucoma in Mongolia. A population-based survey in Hovsgol province, northern Mongolia. Arch Ophthalmol (Chicago, III: 1960). 1996;114(10):1235-41.

5. Foster PJ, Oen FT, Machin D, et al. The prevalence of glaucoma in Chinese residents of Singapore: a cross-sectional population survey of the Tanjong Pagar district. Arch Ophthalmol (Chicago, III: 1960). 2000;118(8):1105-11.

6. Dandona L, Dandona R, Mandal P, et al. Angle-closure glaucoma in an urban population in southern India. The Andhra Pradesh eye disease study. Ophthalmology. 2000;107(9):1710-6.

7. He M, Foster PJ, Ge J, et al. Prevalence and clinical characteristics of glaucoma in adult Chinese: a population-based study in Liwan District, Guangzhou. Invest Ophthalmol Vis Sci. 2006:47(7):2782-8

8. Baskaran M, Foo RC, Cheng CY, et al. The prevalence and types of Glaucoma in an urban Chinese population: the Singapore Chinese eye study. JAMA Ophthalmol. 2015;133(8):874-80.

9. Cheng JW, Zong Y, Zeng YY, et al. The prevalence of primary angle closure glaucoma in adult Asians: a systematic review and meta-analysis. PLoS One. 2014;9(7):e103222.

10. Liu ML, Wang YX. The prevalence of blindness caused by primary angle closure glaucoma in middle-aged Chinese population: a systematic review and meta-analysis. [Zhonghua yan ke za zhi] Chin J Ophthalmol. 2017;53(5):373-7.

11. Congdon NG, Quigley HA, Hung PT, et al. Screening techniques for angleclosure glaucoma in rural Taiwan. Acta Ophthalmol Scand. 1996;74(2):113-9.
12. Alsbirk PH. Primary angle-closure glaucoma. Oculometry, epidemiology, and genetics in a high risk population. Acta Ophthalmol Suppl. 1976;127:5-31.

13. Lowe RF. Aetiology of the anatomical basis for primary angle-closure glaucoma. Biometrical comparisons between normal eyes and eyes with primary angle-closure glaucoma. Br J Ophthalmol. 1970;54(3):161-9.

14. Sihota R, Lakshmaiah NC, Agarwal HC, et al. Ocular parameters in the subgroups of angle closure glaucoma. Clin Exp Ophthalmol. 2000; 28(4):253-8.

15. Congdon $N G$, Youlin $Q$, Quigley $H$, et al. Biometry and primary angle-closure glaucoma among Chinese, white, and black populations. Ophthalmology. 1997;104(9):1489-95.

16. $\mathrm{Ho} \mathrm{H}$, Ozaki M, Mizoguchi T, et al. Angle-closure glaucoma in Asians: comparison of biometric and anterior segment parameters between Japanese and Chinese subjects. Graefes Arch Clin Exp Ophthalmol. 2015; 253(4):601-8.

17. Aung T, Nolan WP, Machin D, et al. Anterior chamber depth and the risk of primary angle closure in 2 East Asian populations. Arch Ophthalmol (Chicago, III: 1960). 2005;123(4):527-32.

18. Hung PT, Chou LH. Provocation and mechanism of angle-closure glaucoma after iridectomy. Arch Ophthalmol (Chicago, III: 1960). 1979;97(10):1862-4.

19. He J, Zou H, Lee RK, et al. Prevalence and risk factors of primary open-angle glaucoma in a city of Eastern China: a population-based study in Pudong New District, Shanghai. BMC Ophthalmol. 2015;15:134.

20. He J, Zou H, Tong $X$, et al. Prevalence of primary glaucoma among adults aged 50 years or above population in Huamu community: a cross-sectional survey in Shanghai, 2011. [Zhonghua yan ke za zhi] Chin J Ophthalmol. 2014;50(5):349-54.

21. Foster PJ, Buhrmann R, Quigley HA, et al. The definition and classification of glaucoma in prevalence surveys. Br J Ophthalmol. 2002;86(2):238-42.

22. Kreuger DE, Milton RC, Maunder LR. The Framingham eye study: introduction to the monograph. Surv Ophthalmol. 1980;24(6):614-20.

23. Wolfs RC, Grobbee DE, Hofman A, et al. Risk of acute angle-closure glaucoma after diagnostic mydriasis in nonselected subjects: the Rotterdam study. Invest Ophthalmol Vis Sci. 1997;38(12):2683-7.

24. Zhao JL. An epidemiological survey of primary angle-closure glaucoma (PACG) in Tibet. [Zhonghua yan ke za zhi] Chin J Ophthalmol. 1990; 26(1):47-50

25. Van Herick W, Shaffer RN, Schwartz A. Estimation of width of angle of anterior chamber. Incidence and significance of the narrow angle. Am 」 Ophthalmol. 1969;68(4):626-9.

26. Alsbirk PH. Limbal and axial chamber depth variations. A population study in Eskimos. Acta Ophthalmol. 1986;64(6):593-600.

27. Thomas R, George T, Braganza A, et al. The flashlight test and van Herick's test are poor predictors for occludable angles. Aust N Z J Ophthalmol. 1996; 24(3):251-6.

28. Cockburn DM. Slitlamp estimate of anterior chamber depth as a predictor of the gonioscopic visibility of the angle structures. Am J Optom Physiol Optic. 1982;59(11):904-8.

29. Foster PJ, Devereux JG, Alsbirk PH, et al. Detection of gonioscopically occludable angles and primary angle closure glaucoma by estimation of limbal chamber depth in Asians: modified grading scheme. Br J Ophthalmol. 2000;84(2):186-92.

30. Baskaran M, Oen FT, Chan YH, et al. Comparison of the scanning peripheral anterior chamber depth analyzer and the modified van Herick grading system in the assessment of angle closure. Ophthalmology. 2007;114(3): 501-6.

31. Kamo J, Saso M, Tsuruta M, et al. Aging effect on peripheral anterior chamber depth in male and female subjects investigated by scanning peripheral anterior chamber depth analyzer. Nippon Ganka Gakkai Zasshi. 2007:111(7):518-25.

32. Mohamed-Noor J, Abd-Salam D. Refractive errors and biometry of primary angle-closure disease in a mixed Malaysian population. Int J Ophthalmol. 2017;10(8):1246-50.

33. Vargas E, Drance SM. Anterior chamber depth in angle-closure glaucoma. Clinical methods of depth determination in people with and without the disease. Arch Ophthalmol (Chicago, III: 1960). 1973;90(6):438-9.

\section{Publisher's Note}

Springer Nature remains neutral with regard to jurisdictional claims in published maps and institutional affiliations. 